\title{
STUDIES ON ON-LINE STATE AND PARAMETER ESTIMATION THROUGH A REAL-TIME PROCESS SIMULATOR
}

\author{
S. Feyo de Azevedo*; P. Pimenta", F. Oliveira ${ }^{*}$ and E.C. Ferreira ${ }^{* * *}$ \\ "Departamento de Engenharia Química, Fac. de Engenharia Univ. do Porto, Rua dos Bragas \\ 4099 Porto Codex PORTUGAL \\ ** University Minho, Engenharia Biológica, Largo do Paço, 4700 Braga PORTUGAL
}

\begin{abstract}
A PC-based simulator of non-linear multiple-input multiple-output dynamic systems was employed for studies concerning the on-line estimation of both 'non-measured' state variables and multiple specific growth rates of a virtual baker's yeast fed-batch fermentation. The identification algorithms were implemented in an 'Observer Computer' which received, optionally in 'real-time', in 'scaled time' or in 'simulation time', the 'measured' state variables generated by the process simulator in the 'Process Computer'. The state observer showed a robust behaviour under different situations where process parameters, operating variables and measuring characteristics (noise and delays) were changed in the process computer. The simulation package was seen to be an efficient and cheap tool for the simulation of non-linear processes with time varying parameters, providing a realistic environment for tests of identification, estimation and control strategies.
\end{abstract}

Keywords. real-time simulator; on-line identification; software sensors; baker's yeast.

\section{INTRODUCTION}

Real processes in the chemical, biochemical and food industry are in their vast majority nonlinear MIMO systems. Their dynamics and control are difficult to study both for theoretical and practical reasons. In many instances experiments with real industrial processes are not carried out for reasons of economy and safety. Often on-line measurements are not available or simply they are too expensive. Advanced control strategies rely on adaptive techniques based on the knowledge of the system state. When experimental observation is lacking, 'software sensors' represent a major, and often the only, alternative to allow optimal process operation to be enforced. The robustness of such sensors needs however to be tested in flexible environments where difficulties such as measurement noises, time delays and loads to the process can be readily implemented on-line. Studies on state and parameters estimation applied to the growth of baker's yeast in fed-batch regime were conducted in one such environment.
The set-up employed is represented in Fig. 1.

The general simulator with the application to the biological system is installed and run in the 'process computer'. Communications are performed via serial RS232 under a protocol which is specific of the simulator. The theoretical approach for state and parameter estimation is implemented in the 'observer computer'.

THE MODEL FOR

BAKER'S YEAST GROWTH

The dynamical model for the fed-batch fermentor was obtained from a mass balance on the components, considering that the reactor was well mixed, the yield coefficients were constant and the dynamics of the gas phase could be neglected. The kinetic model proposed by Sonnleitner and Käppeli (1986) was employed. The set of model equations 
can be written in matrix form as suggested by Dochain (1991) :

$$
\frac{d}{d t}\left(\begin{array}{l}
X \\
S \\
E \\
C \\
G
\end{array}\right)=\left(\begin{array}{ccc}
1 & 1 & 1 \\
-k_{1} & -k_{2} & 0 \\
0 & k_{3} & -k_{4} \\
-k_{5} & 0 & -k_{6} \\
k_{7} & k_{8} & k_{9}
\end{array}\right)\left(\begin{array}{l}
\mu_{S}^{o} \\
\mu_{S}^{r} \\
\mu_{e}^{o}
\end{array}\right) X-D\left(\begin{array}{l}
X \\
S \\
E \\
C \\
G
\end{array}\right)+\left(\begin{array}{c}
0 \\
D S_{i n} \\
0 \\
O T R \\
0
\end{array}\right)-\left(\begin{array}{c}
0 \\
0 \\
0 \\
0 \\
C T R
\end{array}\right)
$$

In the former - $X, S, E, C, G$ - are, respectively, biomass, glucose, ethanol, oxygen and carbon dioxide concentrations; $\mu_{\mathrm{s}}^{0}, \mu_{\mathrm{s}}^{r}$ and $\mu_{\mathrm{e}}^{0}$ represent the specific growth rates for the three pathways proposed by Sonnleitner and Käppeli; $D$ is the dilution rate and the $\mathrm{k}_{\mathrm{i}}(\mathrm{i}=1$ to 9) are the yield coefficients; $S_{i n}$ is the substrate concentration in the feed; OTR is the oxygen transfer rate (defined as OTR $=K_{L} a$ $\left(C^{*}-C\right)$ where $K_{L} a$ is the mass transfer coefficient and $C^{*}$ is the equilibrium concentration of dissolved oxygen) and CTR is the carbon dioxide transfer rate. The dynamics of $\mathrm{CO}_{2}$ may be assumed as being very fast relatively to the dynamics of the other components. Also, the concentration of carbon dioxide at the $\mathrm{pH}$ of operation is known to be low. Hence the balance equation of $\mathrm{CO}_{2}$ can be simplified by assuming $\mathrm{dG} / \mathrm{dt}=0$ and $\mathrm{G}=$ 0 . Defining $Q_{\mathrm{CO} 2}$ as the gas outflow of $\mathrm{CO}_{2}$, this leads to -

$Q_{\mathrm{CO} 2}=C T R=\left(K_{7} \mu_{s}^{o}+K_{8} \mu_{s}^{r}+K_{9} \mu_{e}^{o}\right) X$

The relevant kinetic data were taken from Sonnleitner and Käppeli. The yield coefficients proposed by Pomerleau and Perrier (1990) were employed.

\section{THE DYNAMIC SIMULATOR}

'MIMOSA - Multiple Input Multiple Output Systems Analysis' - is a package which simulates in 'real-time', in 'scaled time' or in 'simulation time' the behaviour of processes represented mathematically by sets of nonlinear differential-algebraic equations (Pimenta and Feyo de Azevedo, 1992a,b). 'Scaled time' is an optional form of operation where one second of real time corresponds to 'scale' seconds of simulation, 'scale' being an userdefined parameter. The option 'simulation time' corresponds to the form of operation where no time control is produced.

The process as simulated by MIMOSA is represented in Fig. 2. The relevant variables for the purpose of process implementation are grouped in six main vectors, viz -

$$
\begin{aligned}
& c[\text { ] - control inputs } \\
& \mathrm{u[} \text { ] - process loads (disturbances) } \\
& \text { ud[ ] - delayed process loads } \\
& \text { p[ ] - process parameters } \\
& y[\text { ] - 'system state variables' } \\
& f[] \text { - derivatives of 'system state } \\
& \text { variables' }
\end{aligned}
$$

The 'system state vector' - $y[$ ] is a concept specific of MIMOSA. It is composed of all variables which have their first derivative explicitly represented in the set of model equations. This includes the formal state variables and all the other variables which are the result of adding to the formal state the dynamic equations for the sensors and final control elements. All system state variables are eligible for output via a D/A interface or an RS232 port, depending on the version of the package being employed. The output is thus constituted by the measured state variables $\mathrm{y}_{\mathrm{m}^{-}}$and the measured loads $\underline{\mathrm{u}}_{\mathrm{m}}$. Both are subsets of $y$.

The general solution of MIMOSA (coded in MS C Vs. 5.1) is based on the integration in the time-domain of the set of differential-algebraic equations which constitute the process model. This is achieved in the current version by employing a variable-step algorithm based on Fehlberg's embedded 4th/5th order, RungeKutta type formulae (Chapra and Canale, 1989).

A basic cycle of work consists on the following tasks - (i) Reads control action; (ii) Proceeds with integration; (iii) Displays and files results; (iv) Outputs measurements; (v) Checks keyboard for interaction while controlling time. Currently, with a 80486 based machine, a wellconditioned set of 20 differential equations can be processed in 0.25 seconds, i. e. an observer can 'see' the process every 0.25 seconds.

The basic concept to create an operator interface was that of 'all-in-one' Graphics Interface where all interactive information is displayed in one single page (screen). This is a major feature for simplicity of operation. 
Figure 3 shows the 'all-in-one' approach. In the upper half of the screen a graphics facility emulating a 4-pen register is available. In the lower half all main variables are monitored and made available for on-line change. In particular it should be noted that :

i. All system state variables y[ ] can be monitored and all are eligible as output variables with a superimposed 'userdefined' random noise;

ii. All parameters named as $\mathrm{p}[\mathrm{]}$ in the model equations can be monitored and their values changed on-line. This includes the noises and dead-times defined in a data file, which MIMOSA automatically will add to the list of parameters and as such will make available for on-line change.

The User creates his own case by writing an ASCII file with the right-hand-side of the system model differential equations, together with the relevant algebraic equations. A data file with information on inputs, system parameters and output characteristics is the other file to be supplied. In constructing both files a few simple rules have to be observed (Pimenta and Feyo de Azevedo, 1992a,b).

With one single command MIMOSA creates a formal case-study (an executable file) which can be run with the data file supplied. During operation, loads to the process can be generated through the keyboard of the process computer. Also, the declared time-varying parameters (including delays and measurement noises) can be changed on-line and in real time. Finally, the appropriate control actions (if it is the case) generated by an external controller (computer or standard industrial system) can be sent to the process.

\section{THE OBSERVER ALGORITHM}

The approach proposed by Bastin and Dochain (1990) was implemented in the 'Observer Computer'. Equation (1) can be written in the form of the so-called 'general dynamical model for stirred tank bioreactors' -

$$
\frac{d \xi}{d t}=K \mu X-D \xi+U
$$

in which: $\xi$ is the vector of the bioprocess components $(\operatorname{dim}(\xi)=N) ; \mu$ is the specific reaction rate vector $(\operatorname{dim}(\mu)=\mathrm{M}) ; K$ is the yield coefficients matrix $(\operatorname{dim}(\mathrm{K})=\mathrm{NxM}) ; U$ is the feed and the gaseous outflow rates vector $(\operatorname{dim}(U)=N)$.

This state space representation can be divided in two partitions: the first one includes the equations relative to the measured state variables $\left(\xi_{1}\right)$; the second partition, the equations relative to the nonmeasured state variables $\left(\xi_{2}\right)$. The dynamical model is rewritten as :

$$
\begin{aligned}
& \frac{d \xi_{1}}{d t}=K_{1} \mu X-D \xi_{1}+U_{1} \\
& \frac{d \xi_{2}}{d t}=K_{2} \mu X-D \xi_{2}+U_{2}
\end{aligned}
$$

where $K_{1}$ (a full rank matrix), $K_{2}, U_{1}, U_{2}=$ division of $K$ and $U$ according to each partition. A transformation is applied to the nonmeasured state variables partition and with the corresponding $Z$ dynamics:

$$
\begin{aligned}
& Z=\xi_{2}-K_{2} K_{1}^{-1} \xi_{1} \\
& \frac{d Z}{d t}=-D Z+U_{2}-K_{2} K_{1}^{-1} U_{1}
\end{aligned}
$$

A "Luenberger-type" asymptotic observer can be written using eq. (7) with $Z$ replaced by its estimate $\hat{Z}$ and the non-measured state variables estimated by the following equation:

$\hat{\xi}_{2}=\hat{Z}+K_{2} K_{1}^{-1} \xi_{1}$

For the specific growth rates the following estimator is employed:

$$
\begin{aligned}
& \frac{d \hat{\psi}}{d t}=\hat{\mu} X-D \psi+K_{1}^{-1} U_{1}+\omega(\psi-\hat{\psi}) X \\
& \frac{d \hat{\mu}}{d t}=\gamma(\psi-\hat{\psi}) X
\end{aligned}
$$

with $\psi=K_{1}^{-1} \xi_{1}$, a transformation to decouple the equations with respect to the specific growth rate; $\omega, \gamma$ are diagonal matrices containing time varying tuning parameters updated by a pole placement procedure (Pomerleau and Perrier, 1990). A discrete version of the estimator algorithms was 
implemented by first order Euler approximation with a sampling period of 6 minutes.

\section{RESULTS AND CONCLUSIONS}

Two case studies illustrate the application. In both, the initial conditions were -

$$
\begin{aligned}
& X(0)=0.1 \mathrm{~g} / \mathrm{l}, \quad S(0)=0.8 \mathrm{~g} / \mathrm{l}, \mathrm{E}(0)=0 \mathrm{~g} / \mathrm{l}, \\
& \mathrm{C}(0)=0.0066 \mathrm{~g} / \mathrm{l}, \mathrm{V}(0)=3.5 \mathrm{l}, \mathrm{S}_{\mathrm{in}}(0)=5 \mathrm{~g} / \mathrm{l} .
\end{aligned}
$$

The value for $K_{L}$ a was assumed as $60 \mathrm{hr}^{-1}$.

In the first a constant dilution rate of $0.04 \mathrm{hr}^{-1}$ is assumed. The final volume of $10 \mathrm{I}$ is taken as criterion to stop the feeding. Figure 3 represents the process simulation with MIMOSA.

Glucose, dissolved oxygen and ethanol were the measured variables available to the 'observer'. After about 3 hours a white noise of approximately $5 \%$ was imposed on the measurement of oxygen. The dotted lines in Fig. 4 represent the biomass and the flux of $\mathrm{CO}_{2}$ estimated. The continuous lines represent the 'true' values obtained with the simulator. The reconstruction of the relevant specific growth rates is shown in Fig. 5. The influence of noise is visible in the figures, but it is well dumped by the filter.

In the second case study an exponential evolution of the dilution rate $[D(t)=0.03$ $\exp (0.1 t)]$ was imposed in MIMOSA. A white noise with variance 0.01 was considered in the measurement of ethanol. Figure 6 shows the observed (dotted lines) and the 'true' values of biomass, glucose and $\mathrm{CO}_{2}$ outflow obtained from single measurements of dissolved $\mathrm{O}_{2}$ and ethanol. Here, a simplification had to be performed, having neglected the oxidative specific growth rate on ethanol, $\mu_{\mathrm{e}}$. The 'Observer' is not sensitive to the noise imposed and performs well.

Further analysis is beyond the scope of this paper. The 'process' simulator provides, as described, a realistic environment for on-line test of identification strategies. Its User interaction capabilities, such as imposing dead times and noises, imposing on-line variation of parameters and switching between real-time, scaled time and simulation time, are well above those allowed by other packages available. The setup proposed constitutes thus, a PC based laboratory for research and development in process control.

\section{ACKNOWLEDGEMENTS}

This work was partially supported by JNICT Junta Nacional de Investigação Científica e Tecnológica, under contract numbers BD/224/90-IF and BD/1476/91-RM and INIC Instituto Nacional de Investigação Científica.

\section{LITERATURE CITED}

Bastin, G. and Dochain, D. (1990). On-Line Estimation and Adaptive Control of Bioreactors. Elsevier Science Publishers, Amsterdam.

Chapra, S. C. and Canale, R. (1988). Numerical Methods for Engineers. 2nd Ed., McGraw-Hill International Editions, Applied Mathematics Series, New York.

Dochain, D. (1991). Design of Adaptive Controlers for Non-linear Stirred Tank Bioreactors: Extension to the MIMO Situation. J. Proc. Cont., 1:1, 41-48.

Pimenta, P. and Feyo de Azevedo, S. (1992). Real Time Dynamic Simulation of Nonlinear MIMO Systems. European Symp. on Comp. App. in Proc. Eng. (accepted)

Pimenta, P. and Feyo de Azevedo, S. (1992). MIMOSA User's Manual and Applications Reference Book. V. 1.0, Internal Publication (in English), Departamento de Engenharia Química, Faculdade de Engenharia, Universidade do Porto.

Pomerleau, Y. and Perrier, M. (1990). Estimation of Multiple Specific Growth Rates in Bioprocesses. A.I.Ch.E. J., 36:2, 207-215.

Sonnleitner, B. and Käppeli, O. (1986). Growth of Saccharomyces Cerevisiae is Controlled by its Limited Respiratory Capacity: Formulation and Verification of a Hypothesis. Biotechnol. Bioeng., 28, 927-937. 


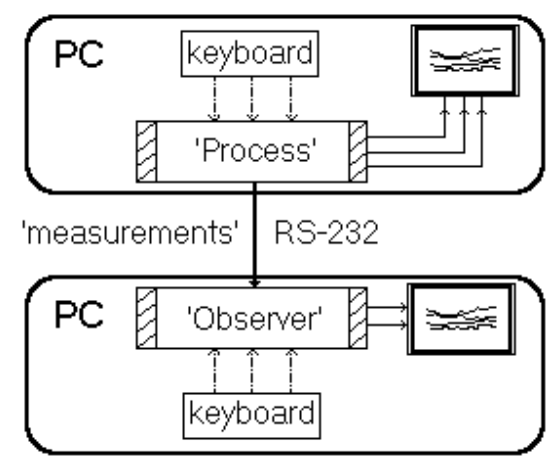

Fig. 1. Laboratory set-up.

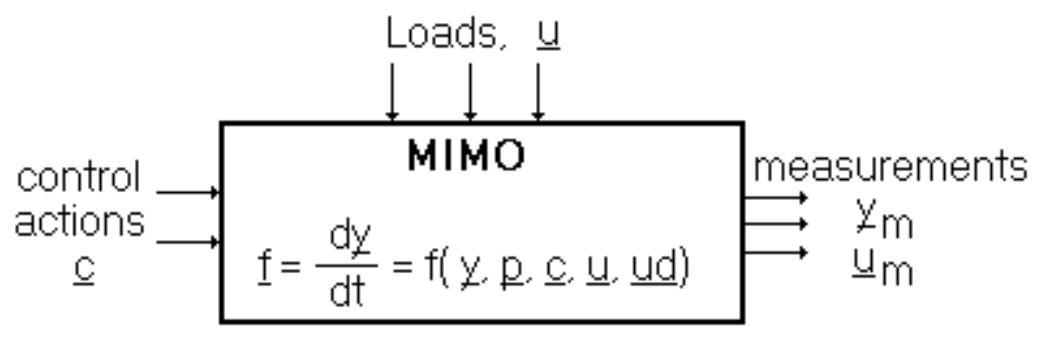

Fig. 2. Representation of a MIMO process.

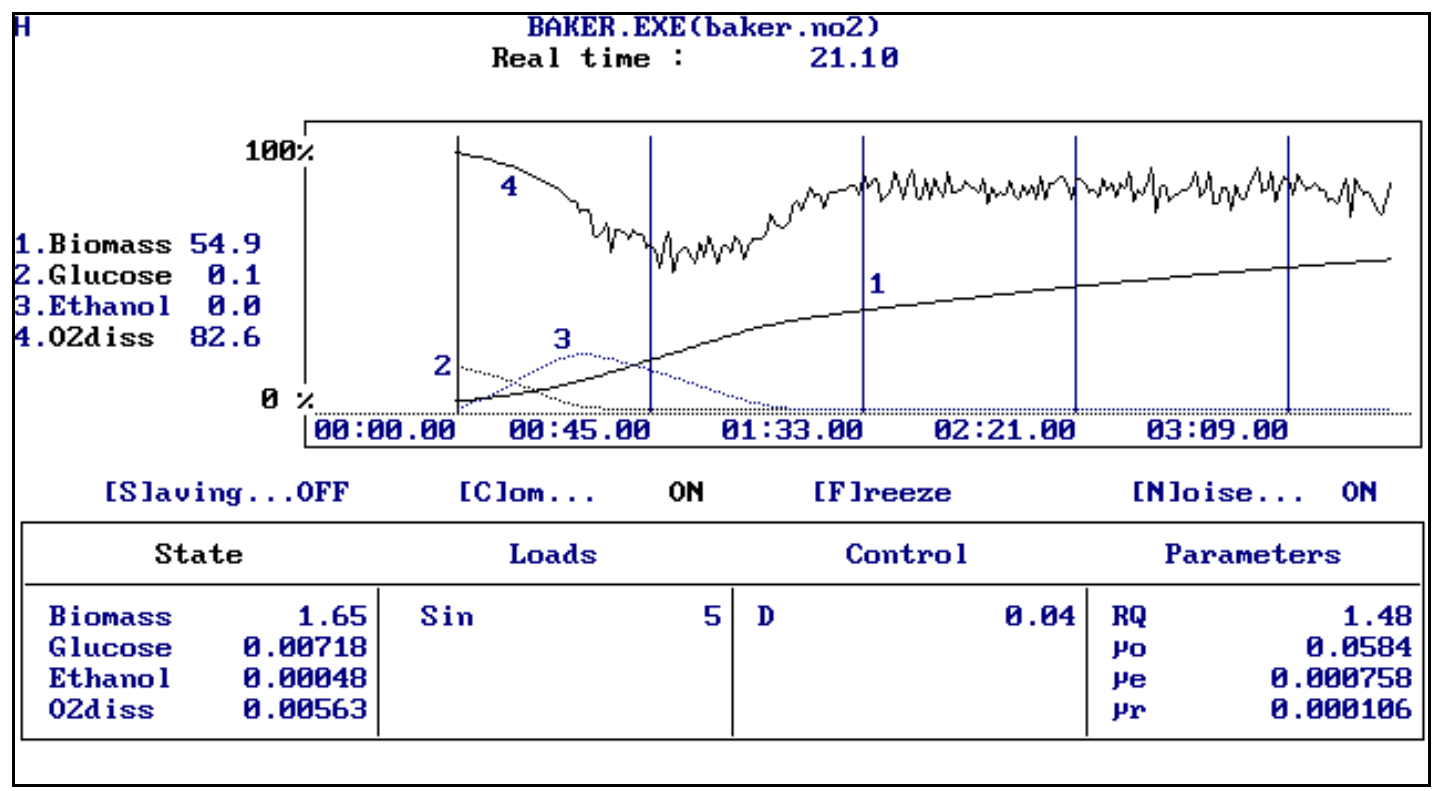

Fig. 3. Process monitoring in MIMOSA. 


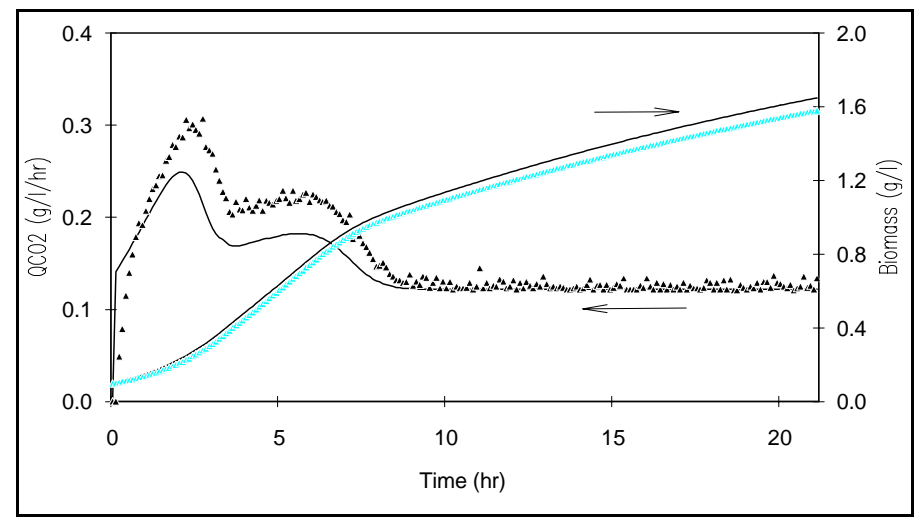

Fig. 4. 'Observed' - (dotted lines) vs. 'true' state properties (continuous line).

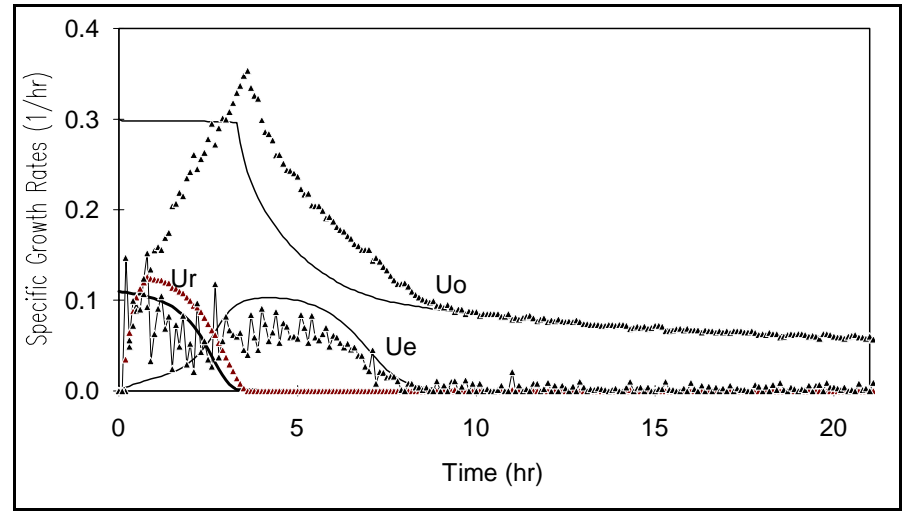

Fig. 5. 'Observed' (dotted lines) vs. 'true' specific growth rates (continuous line).

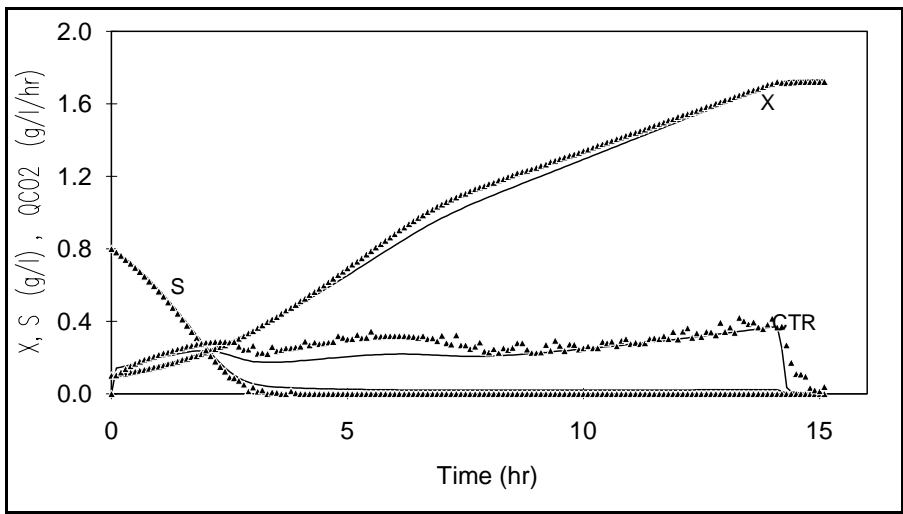

Fig. 6. Estimation of biomass, glucose and outflow of $\mathrm{CO}_{2}$ from measurements of ethanol and dissolved oxygen. 